\title{
Correction: MicroRNA-34a expression levels in serum and intratumoral tissue can predict bone metastasis in patients with hepatocellular carcinoma
}

\author{
Zuo-Lin Xiang ${ }^{1, *}$, Xiao-Mei Zhao ${ }^{1, *}$, Li Zhang ${ }^{1}$, Ping Yang ${ }^{1}$, Jia Fan ${ }^{2}$, Zhao-You Tang ${ }^{2}$, \\ Zhao-Chong Zeng ${ }^{1}$ \\ ${ }^{1}$ Department of Radiation Oncology, Zhongshan Hospital, Fudan University, Shanghai, China \\ ${ }^{2}$ Department of Liver Cancer Institute, Zhongshan Hospital, Fudan University, Shanghai, China \\ *These authors have contributed equally to this work
}

Published: October 29, 2019

Copyright: Xiang et al. This is an open-access article distributed under the terms of the Creative Commons Attribution License 3.0 (CC BY 3.0), which permits unrestricted use, distribution, and reproduction in any medium, provided the original author and source are credited.

This article has been corrected: Due to errors in image processing, there were misplaced images in Figure 2. An updated Figure 2 using the original data is shown below. The authors declare that these corrections do not change the results or conclusions of this paper.

Original article: Oncotarget. 2016; 7:87246-87256. https://doi.org/10.18632/oncotarget.13531 
A

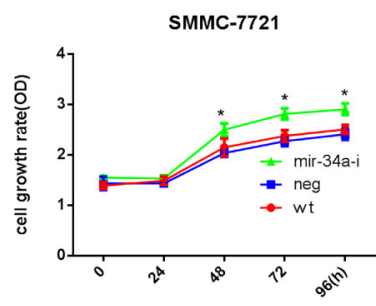

B

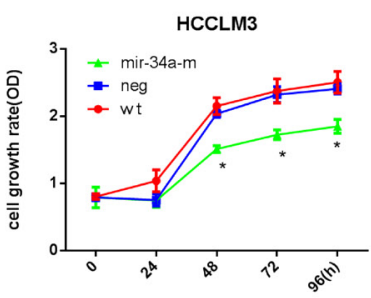

C

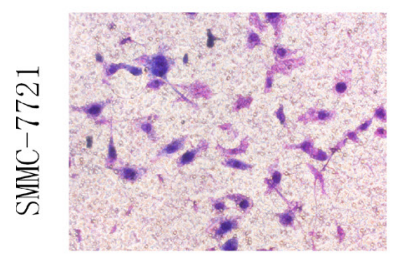

WT

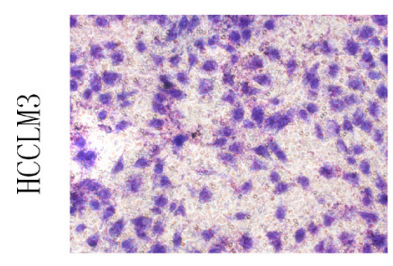

NG

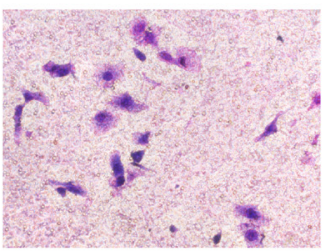

NG

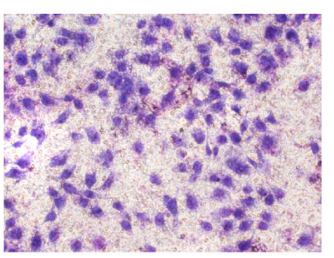

NG

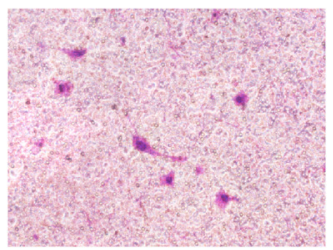

NG

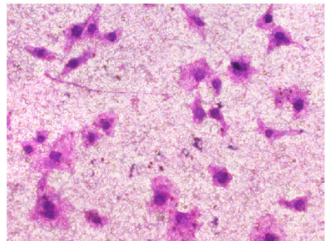

miR-34a-i

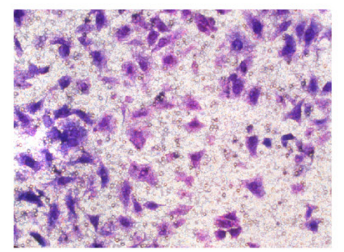

miR-34a-m

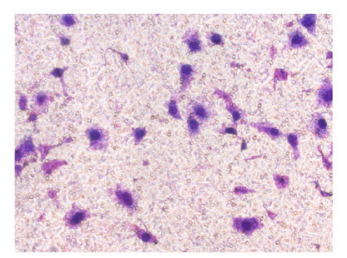

D

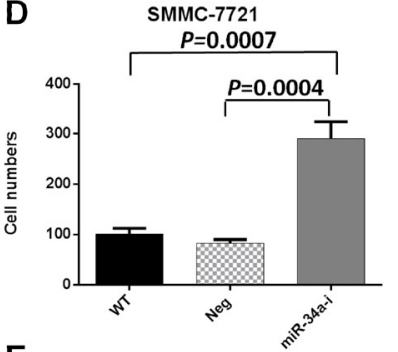

E

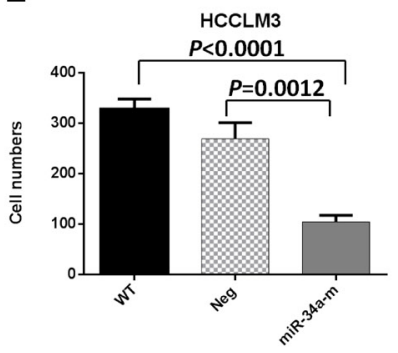

G
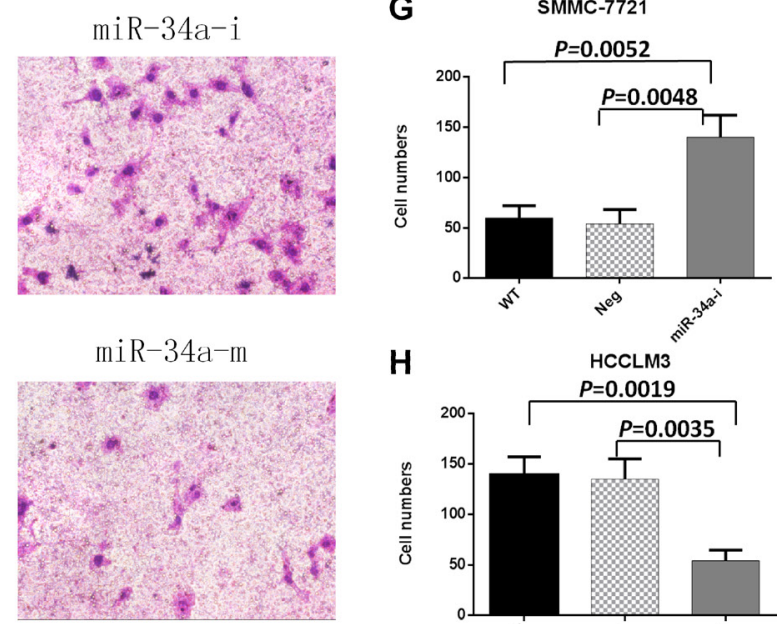

H
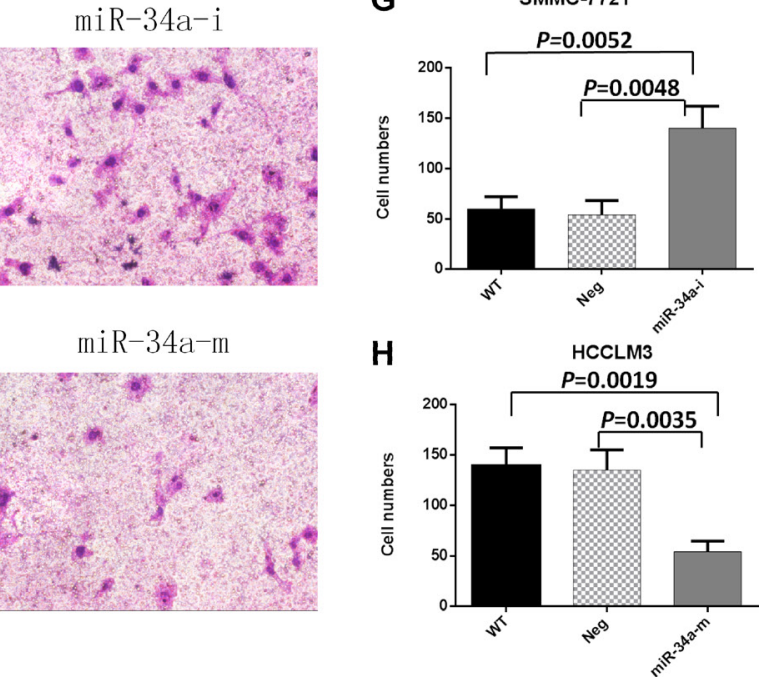

Figure 2: Proliferation, migration, and invasion assays of SMMC-7721 and HCCLM3 cells after transfection with the indicated oligonucleotides. WT, NG, miR-34a-m, and miR-34a-i indicate non-transfected, negative control oligonucleotide-transfected, miR-34a mimic- transfected, and miR-34a inhibitor-transfected cells, respectively. (A) SMMC-7721 cell growth rates after transfection with the miR-34a inhibitor. (B) HCCLM3 cell growth rates after transfection with the miR-34a mimic. ${ }^{*}=P<0.05$, compared to negative controls at the same time point. (C) Representative images of the migration assay. (D) and (E) Numbers of migrating cells on the undersides of the membranes. Data are presented as mean \pm SEM and are representative of three independent experiments. The images were acquired at a magnification of 200×. (F) Representative images of the invasion assay. (G) and (H) Numbers of invading cells on the undersides of the membranes. Data are presented as mean \pm SEM and are representative of three independent experiments. The images were acquired at a magnification of $200 \times$. 